\title{
58. INTERACTION BETWEEN SEDIMENT AND INTERSTITIAL WATER NEAR THE JAPAN TRENCH, LEG 57, DEEP SEA DRILLING PROJECT
}

\author{
George W. Moore, U.S. Geological Survey, Menlo Park, California \\ and \\ Joris M. Gieskes, Scripps Institution of Oceanography, La Jolla, California
}

\begin{abstract}
Analyses of water samples taken by means of an in-hole sampler generally show good agreement with analyses of samples collected by routine shipboard squeezing techniques. At Sites 438 and 439 , a decrease in salinity with depth is related to former freshwater flow from an aquifer that crops out at an anticline on a deep sea terrace between Japan and the top of the trench slope of the Japan Trench. This former subaerial recharge suggests significant late Cenozoic subsidence of the terrace, because it now lies at a water depth of 1500 meters. Samples from the trench slope at Site 440 have extremely high values of alkalinity and ammonia, presumably because of a favorable combination of high sedimentation rate and organic carbon content. Diagenetic conditions on the trench slope favor formation of the $\mathrm{Fe}-\mathrm{Mg}$ carbonate mineral, ankerite; at Site 440 it first occurs at a depth below the sea floor of only 29 meters in late Pleistocene strata. Undissolved diatoms persist to relatively great depth at the sites of Leg 57 because of a low geothermal gradient caused by subduction. Secondary silica lepispheres first appear at 851 meters at the most landward and warmest site, Site 438, in strata 16 million years old with an ambient temperature of $31^{\circ} \mathrm{C}$.
\end{abstract}

\section{SAMPLING AND ANALYTICAL PROCEDURES}

During Leg 57 (Figure 1), samples of interstitial water were collected at all of the sites by standard shipboard squeezing techniques and at Sites 438 and 440 by means of the IPOD in-hole sampler, which collects both water for chemical analysis in a stainless steel tubular coil and water for gas analysis in a connected copper coil (Barnes et al., 1979). The in-hole sampler was used to a depth below the sea floor of 449 meters at Site 438 and 235 meters at Site 440. In this chapter we present results of shipboard chemical analyses and subsequent analyses carried out in the laboratory of J. M. Gieskes at Scripps Institution of Oceanography. Analytical methods follow those described by Gieskes (1974), with minor modifications.

Shipboard in-hole data on $\mathrm{pH}$, alkalinity, chlorinity, calcium, and magnesium (except $\mathrm{Mg}$ from Site 438) generally show good agreement with the samples obtained by routine shipboard squeezing techniques (Tables 1 and 2; Figures 2 and 3 ). The shipboard results for the inhole samples of Site 438 show large differences for magnesium between these in situ samples and those obtained from routine shipboard squeezing methods. We reanalyzed in-hole sample splits stored in copper tubes provided by Dr. R. Barnes by atomic absorption spectroscopy to investigate this discrepancy. The new results indicate good agreement with shipboard squeezings. Thus it is possible that the shipboard results for magnesium from the in-hole sampler are in error, although no reason is apparent to explain the discrepancy.

The results for ammonia and silica were obtained from the overflow water of the in-hole sampler and had to be corrected for dilution by distilled water. Agreement for Site 438 with routinely collected samples is good (Figure 2), but for Site 440 the agreement is poor. This can be explained by larger amounts of copper in the overflow water due to the corrosive nature of the high-alkalinity pore water at Site 440 . Copper ions interfere with the colorimetric determination of ammonia and silica.

In general, however, we conclude that the in-hole samples essentially confirm the validity of the interstitial water sample data collected by routine squeezing, even for the minor constituents, silica and ammonia. This is in agreement with the conclusions of Barnes and others (1979).

\section{SALINITY DECREASE WITH DEPTH}

An interesting phenomenon at Sites 438 and 439 (water depth about $1600 \mathrm{~m}$ ) is a downhole decrease in salinity and chlorinity. The chlorinity at the deepest sample at Site 438 is only about 50 per cent of that of seawater. These data imply that fresh water has been advected to the sites from an outcrop area.

A possible source of fresh water is through sandstone and conglomerate layers in the deeper strata of the sites. Because boulders in the conglomerate are very large, 


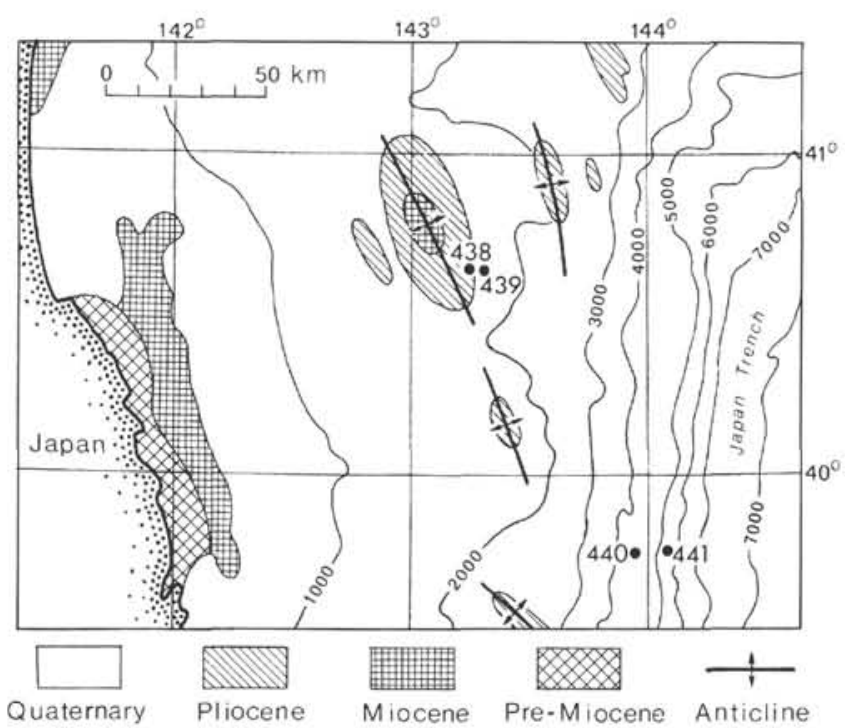

Figure 1. Offshore geologic map of the area drilled during DSDP Leg 57, showing the position of the drill sites in relation to the Japan Trench and to breached anticlines on the deep sea terrace lafter Honza et al., 1978). Submarine contours in meters.

however, both conglomerate and sandstone are probably local deposits and do not extend all the way to the shoreline $150 \mathrm{~km}$ to the west. Therefore the freshwater recharge area is ancient - probably a submerged outcrop area near the sites.

The submarine geologic map shows an anticlinal area of Miocene and Pliocene sea floor outcrops with an area of about $1000 \mathrm{~km}^{2}$ centered about $20 \mathrm{~km}$ northwest of the sites (Figure 1). Data from reflection profiles and dredged rocks indicate that erosion continued until about the end of the Pliocene (Honza et al., 1978). At the present time, the deep sea terrace, including the recharge area, lies at a water depth of about 1500 meters. The measured salinity gradient at Sites 438 and 439 , which records fossil conditions, shows that the erosion was subaerial and hence that very rapid subsidence has been occurring in this area above the Japan Trench since the freshwater recharge ceased.

\section{HIGH ALKALINITY AND AMMONIA}

Site 438 and adjacent Site 439 consist mainly of clayey-sandy sediment with an appreciable content of diatom remains. Organic carbon content averages 0.5 per cent, and sulfate reduction is essentially complete within the first 100 meters of sediment. Methane production is important at this site. This process is accompanied by the production of ammonia, leading to an ammonia maximum of about $4 \mathrm{mM}$ at about 200 meters.

As a result of the large increase in alkalinity, calcium carbonate precipitates, as reflected in a minimum dissolved calcium at about 100 meters. Dissolved magnesium appears to be consumed throughout the sediment column, in part by uptake in silicate minerals that form during silica diagenesis (Johnson, 1976). Identification of such newly formed silicate minerals is hampered, however, because of high dilution by similar detrital minerals.

Site 440 (water depth $4509 \mathrm{~m}$ ) is characterized by a rapidly deposited Pleistocene sediment section, with a deposition rate of about $20 \mathrm{~cm} / 1000$ years. Dissolved sulfate is depleted within the upper 50 meters of sediment, and below this level diagenesis leads to high levels of methane in core gas pockets. The sulfate reduction is accompanied by very high alkalinity values, up to 92 $\mathrm{meq} / \mathrm{L}$. These high values must be partly a result of ammonia formation during the biogenic methane production processes. A maximum of $8.2 \mathrm{mM} \mathrm{NH}_{4}{ }^{+}$is observed to coincide with this alkalinity maximum. If one assumes that alkalinity produced during sulfate reduction, when properly corrected for calcium carbonate precipitation, is about $40 \mathrm{meq} / \mathrm{L}$, it follows that at least $52 \mathrm{meq} / \mathrm{L}$ of ammonia must have been produced during the methane production processes. If only $8 \mathrm{mM}$ of $\mathrm{NH}_{4}{ }^{+}$remains in the pore fluids, about $32 \mathrm{meq} / \mathrm{L}$ of ammonia must have been lost, perhaps on clay mineral exchange sites, as has been found to occur in reduced sediment by Hartmann and others (1973). The most likely ions to be displaced are $\mathrm{Na}^{+}, \mathrm{K}^{+}$, and $\mathrm{Mg}^{2+}$ (Sayles and Mangelsdorf, 1977). Such exchange with $\mathrm{NH}_{4}{ }^{+}$is suggested by the increase in concentration of dissolved $\mathrm{Mg}$ in the upper 200 meters at Site 440. Unfortunately we could not obtain reliable data on dissolved sodium and potassium to confirm this interpretation, but a comparison with results from rapidly deposited radiolarian-rich nanno ooze at Site 262 in the Timor Trough (Cook, 1974) suggests that sodium and potassium, in addition to magnesium, must also be released to the interstitial water. As a result of these exchange processes and the formation of bicarbonate, the total dissolved solids (Salinity, Table 1) increases with depth in the upper section of Site 440. This increase also was observed by Cook (1974) at Site 262.

Observations of such high alkalinity and ammonia content are relatively rare in marine sediments and thus far have been made at only two DSDP sites -262 and 440. A unique combination of high sedimentation rate and relatively high organic carbon content (associated with microfossil ooze) appears to be required to maintain the alkalinity and ammonia values at such high levels. At lower sedimentation rates, alkalinity and ammonia tend to decrease or be absent because of diffusional exchange with the overlying and underlying sediment layers (Gieskes, 1975).

\section{SECONDARY CALCITE AND ANKERITE}

The conditions which led to the high alkalinity at shallow depth below the sea floor at Site 440 also led to the formation of fine-grained chalky secondary carbonate minerals as identified by X-ray diffraction (Table 3 ). The most notable mineral in this assemblage is ankerite, $\mathrm{Ca}(\mathrm{Fe}, \mathrm{Mg})\left(\mathrm{CO}_{3}\right)_{2}$. Ankerite generally forms in a reducing environment where iron activity is high; for example, it commonly exists as veins in the cleat of coal beds (Soong and Gluskoter, 1977). Boles (1978) has found it in pyrite-bearing Eocene marine sedimentary rocks in Texas. 
TABLE 1

Chemistry of Shipboard Squeezed Samples

\begin{tabular}{|c|c|c|c|c|c|c|c|c|c|c|}
\hline $\begin{array}{c}\text { Sample } \\
\text { (Interval in } \mathrm{cm} \text { ) }\end{array}$ & $\begin{array}{l}\text { Depth } \\
\text { (m) }\end{array}$ & $\mathrm{pH}$ & $\begin{array}{r}\text { Alkalinity } \\
(\mathrm{meq} / \mathrm{L})\end{array}$ & $\begin{array}{l}\text { Salinity } \\
(\%)\end{array}$ & $\begin{array}{c}\mathrm{Cl} \\
(\%)\end{array}$ & $\begin{array}{c}\mathrm{Ca} \\
(\mathrm{mM})\end{array}$ & $\underset{(\mathrm{mM})}{\mathrm{Mg}}$ & $\begin{array}{l}\mathrm{SO}_{4} \\
(\mathrm{mM})\end{array}$ & $\begin{array}{c}\mathrm{NH}_{4} \\
(\mathrm{mM})\end{array}$ & $\begin{array}{l}\mathrm{SiO}_{2} \\
(\mathrm{mM})\end{array}$ \\
\hline \multicolumn{11}{|l|}{ Hole 438} \\
\hline $\begin{array}{l}2-5,140-150 \\
7-5,140-150 \\
11-5,140-150\end{array}$ & $\begin{array}{l}13 \\
60 \\
98\end{array}$ & $\begin{array}{l}7.8 \\
7.6 \\
7.9\end{array}$ & $\begin{array}{l}11.7 \\
17.0 \\
15.3\end{array}$ & $\begin{array}{l}33.8 \\
32.7 \\
32.3\end{array}$ & $\begin{array}{l}19.0 \\
18.6 \\
18.4\end{array}$ & $\begin{array}{l}6.5 \\
2.7 \\
3.0\end{array}$ & $\begin{array}{l}52.5 \\
33.0 \\
33.0\end{array}$ & $\begin{array}{c}13.8 \\
- \\
-\end{array}$ & $\begin{array}{l}0.75 \\
2.57 \\
3.50\end{array}$ & $\begin{array}{l}0.590 \\
0.657 \\
0.620\end{array}$ \\
\hline \multicolumn{11}{|l|}{ Hole 438A } \\
\hline $\begin{array}{l}1-1,140-150 \\
5-4,140-150^{a} \\
11-5,140-150 \\
16-5,88-98 \\
21-7,35-45 \\
26-6,140-150 \\
31-5,140-150 \\
36-5,140-150 \\
41-5,140-150 \\
46-6,140-150 \\
50-5,140-150 \\
56-5,140-150 \\
64-4,140-150 \\
70-5,140-150 \\
79-3,140-150 \\
84-1,140-150\end{array}$ & $\begin{array}{r}1 \\
57 \\
162 \\
208 \\
258 \\
305 \\
352 \\
399 \\
447 \\
497 \\
534 \\
591 \\
665 \\
724 \\
806 \\
851\end{array}$ & $\begin{array}{l}8.1 \\
7.6 \\
7.6 \\
7.4 \\
7.9 \\
7.7 \\
7.8 \\
8.0 \\
8.0 \\
8.0 \\
8.1 \\
8.0 \\
- \\
8.0 \\
7.9 \\
-\end{array}$ & $\begin{array}{r}4.1 \\
9.7 \\
21.3 \\
23.5 \\
21.2 \\
18.2 \\
17.2 \\
14.5 \\
17.6 \\
14.2 \\
13.3 \\
11.8 \\
- \\
7.5 \\
8.2 \\
-\end{array}$ & $\begin{array}{c}34.6 \\
34.6 \\
- \\
32.6 \\
31.3 \\
30.1 \\
30.2 \\
27.8 \\
27.5 \\
27.2 \\
27.5 \\
26.6 \\
27.1 \\
26.6 \\
25.4 \\
26.5\end{array}$ & $\begin{array}{l}19.2 \\
19.2 \\
18.4 \\
18.9 \\
17.6 \\
16.9 \\
17.0 \\
19.0 \\
15.4 \\
15.1 \\
14.7 \\
14.6 \\
15.0 \\
14.5 \\
13.5 \\
14.5\end{array}$ & $\begin{array}{l}9.9 \\
6.6 \\
7.4 \\
8.9 \\
9.6 \\
8.7 \\
8.5 \\
9.4 \\
7.6 \\
7.9 \\
7.3 \\
7.1 \\
6.7 \\
9.6 \\
9.6 \\
9.1\end{array}$ & $\begin{array}{r}52.7 \\
54.0 \\
34.7 \\
32.8 \\
25.3 \\
22.4 \\
25.0 \\
16.1 \\
14.9 \\
12.8 \\
13.0 \\
11.2 \\
13.8 \\
7.4 \\
5.6 \\
10.9\end{array}$ & $\begin{array}{l}27.6 \\
19.0 \\
- \\
0.0 \\
- \\
0.0 \\
- \\
- \\
- \\
- \\
0.0 \\
- \\
- \\
- \\
- \\
0.3\end{array}$ & $\begin{array}{l}0.20 \\
1.45 \\
3.90 \\
4.21 \\
4.12 \\
4.02 \\
1.50 \\
3.40 \\
3.45 \\
3.32 \\
3.19 \\
0.75 \\
3.05 \\
2.73 \\
2.20 \\
2.29\end{array}$ & $\begin{array}{l}0.411 \\
0.477 \\
0.495 \\
0.765 \\
0.659 \\
0.775 \\
0.633 \\
0.723 \\
0.675 \\
1.151 \\
0.583 \\
0.678 \\
0.717 \\
0.661 \\
0.764 \\
0.575\end{array}$ \\
\hline \multicolumn{11}{|l|}{ Hole 438B } \\
\hline $\begin{array}{l}4-6,140-150 \\
9-1,140-150 \\
17-1,140-150\end{array}$ & $\begin{array}{l}862 \\
901 \\
977\end{array}$ & $\begin{array}{c}8.2 \\
- \\
-\end{array}$ & $\begin{array}{l}6.8 \\
- \\
-\end{array}$ & $\begin{array}{l}23.6 \\
22.1 \\
22.8\end{array}$ & $\begin{array}{r}11.4 \\
9.7 \\
9.9\end{array}$ & $\begin{array}{l}12.4 \\
10.8 \\
11.4\end{array}$ & $\begin{array}{l}5.5 \\
3.3 \\
4.1\end{array}$ & $\begin{array}{l}- \\
- \\
-\end{array}$ & $\begin{array}{c}1.35 \\
0.97 \\
-\end{array}$ & $\begin{array}{c}0.556 \\
- \\
0.662\end{array}$ \\
\hline \multicolumn{11}{|l|}{ Hole 439} \\
\hline $\begin{array}{l}7-3,140-150 \\
13-2,140-150 \\
18-1,140-150 \\
30-3,140-150\end{array}$ & $\begin{array}{r}873 \\
928 \\
965 \\
1082\end{array}$ & $\begin{array}{l}- \\
- \\
-\end{array}$ & $\begin{array}{l}- \\
- \\
-\end{array}$ & $\begin{array}{l}22.2 \\
21.8 \\
22.4 \\
19.5\end{array}$ & $\begin{array}{l}12.7 \\
12.9 \\
12.7 \\
11.3\end{array}$ & $\begin{array}{r}8.8 \\
10.2 \\
10.6 \\
9.8\end{array}$ & $\begin{array}{l}5.5 \\
4.9 \\
6.1 \\
3.8\end{array}$ & $\begin{array}{l}- \\
- \\
- \\
-\end{array}$ & $\begin{array}{l}- \\
- \\
-\end{array}$ & $\begin{array}{l}0.552 \\
0.278 \\
0.513 \\
0.068\end{array}$ \\
\hline \multicolumn{11}{|l|}{ Hole 440} \\
\hline $\begin{array}{l}1-3,140-150 \\
6-4,140-150 \\
7-2,130-150\end{array}$ & $\begin{array}{r}5 \\
50 \\
57\end{array}$ & $\begin{array}{l}7.9 \\
7.9 \\
7.6\end{array}$ & $\begin{array}{l}23.8 \\
84.4 \\
88.3\end{array}$ & $\begin{array}{l}35.5 \\
37.8 \\
38.1\end{array}$ & $\begin{array}{l}19.4 \\
19.5 \\
19.8\end{array}$ & $\begin{array}{l}6.7 \\
3.7 \\
3.6\end{array}$ & $\begin{array}{l}52.3 \\
57.9 \\
59.2\end{array}$ & $\begin{array}{l}7.2 \\
- \\
0.0\end{array}$ & $\begin{array}{l}1.22 \\
7.82 \\
8.37\end{array}$ & $\begin{array}{l}0.420 \\
0.527 \\
0.567\end{array}$ \\
\hline \multicolumn{11}{|l|}{ Hole 440A } \\
\hline $\begin{array}{l}2-5,140-150 \\
7-6,140-150\end{array}$ & $\begin{array}{r}90 \\
138\end{array}$ & $\begin{array}{l}7.7 \\
8.1\end{array}$ & $\begin{array}{l}91.8 \\
86.2\end{array}$ & $\begin{array}{l}38.1 \\
37.8\end{array}$ & $\begin{array}{l}19.5 \\
19.5\end{array}$ & $\begin{array}{l}2.8 \\
2.7\end{array}$ & $\begin{array}{l}60.9 \\
58.4\end{array}$ & $\overline{1.6}$ & $\begin{array}{l}8.00 \\
7.23\end{array}$ & $\begin{array}{l}0.453 \\
0.480\end{array}$ \\
\hline \multicolumn{11}{|l|}{ Hole 440B } \\
\hline $\begin{array}{l}4-3,130-150 \\
10-6,130-150 \\
15-5,130-150 \\
20-4,130-150 \\
28-4,0-15 \\
33-3,130-150 \\
40-3,130-150 \\
46-3,130-150 \\
51-4,0-12 \\
56-2,130-150 \\
62-2,0-12 \\
67-2,130-150\end{array}$ & $\begin{array}{l}172 \\
234 \\
280 \\
326 \\
400 \\
448 \\
515 \\
571 \\
619 \\
665 \\
721 \\
769\end{array}$ & $\begin{array}{l}7.8 \\
7.8 \\
8.4 \\
8.3 \\
7.8 \\
- \\
7.7 \\
- \\
8.4 \\
\overline{8.5} \\
-\end{array}$ & $\begin{array}{c}75.3 \\
68.0 \\
61.8 \\
50.4 \\
37.4 \\
- \\
25.8 \\
-\overline{0} \\
20.1 \\
-\overline{4} .1 \\
-\end{array}$ & $\begin{array}{l}37.3 \\
36.3 \\
37.2 \\
35.5 \\
34.2 \\
34.1 \\
32.8 \\
33.8 \\
33.4 \\
31.9 \\
33.6 \\
33.1\end{array}$ & $\begin{array}{l}19.3 \\
19.0 \\
19.7 \\
18.9 \\
18.9 \\
19.4 \\
19.6 \\
19.4 \\
19.6 \\
19.5 \\
19.3 \\
19.7\end{array}$ & $\begin{array}{l}3.6 \\
3.6 \\
4.5 \\
4.7 \\
3.7 \\
3.8 \\
3.6 \\
6.0 \\
6.3 \\
7.2 \\
8.4 \\
8.6\end{array}$ & $\begin{array}{l}62.3 \\
55.4 \\
50.9 \\
44.4 \\
35.9 \\
33.4 \\
30.9 \\
29.2 \\
30.3 \\
26.9 \\
29.1 \\
17.5\end{array}$ & $\begin{array}{l}0.0 \\
0.5 \\
- \\
- \\
- \\
- \\
- \\
- \\
- \\
- \\
- \\
-\end{array}$ & $\begin{array}{l}6.47 \\
6.28 \\
5.02 \\
5.49 \\
5.41 \\
4.95 \\
4.25 \\
4.60 \\
4.97 \\
4.54 \\
3.60 \\
4.03\end{array}$ & $\begin{array}{l}0.529 \\
0.530 \\
0.465 \\
0.485 \\
0.448 \\
0.533 \\
0.685 \\
0.482 \\
0.479 \\
0.363 \\
0.527 \\
0.477\end{array}$ \\
\hline \multicolumn{11}{|l|}{ Hole 441} \\
\hline $1-1,140-150$ & 1 & 7.5 & 2.9 & 35.8 & 19.2 & 10.6 & 52.7 & - & 0.27 & 0.260 \\
\hline \multicolumn{11}{|l|}{ Hole 441A } \\
\hline $\begin{array}{l}5-3,140-150 \\
14-2,0-12\end{array}$ & $\begin{array}{l}390 \\
645\end{array}$ & $\begin{array}{l}- \\
-\end{array}$ & - & $\begin{array}{l}33.3 \\
32.9\end{array}$ & $\begin{array}{l}19.7 \\
19.4\end{array}$ & $\begin{array}{l}14.4 \\
13.9\end{array}$ & $\begin{array}{l}13.3 \\
20.4\end{array}$ & $\begin{array}{l}- \\
-\end{array}$ & $\begin{array}{l}2.62 \\
2.09\end{array}$ & $\begin{array}{l}0.277 \\
0.223\end{array}$ \\
\hline \multicolumn{11}{|l|}{ Hole $441 B^{b}$} \\
\hline $\begin{array}{l}\mathrm{H} 1-3,130-150 \\
\mathrm{H} 2-4,130-150\end{array}$ & $\begin{array}{l}388-401 \\
408-666\end{array}$ & $\begin{array}{l}7.9 \\
8.3\end{array}$ & $\begin{array}{l}8.7 \\
7.3\end{array}$ & $\begin{array}{l}32.0 \\
31.9\end{array}$ & $\begin{array}{l}18.8 \\
18.6\end{array}$ & $\begin{array}{l}12.5 \\
11.5\end{array}$ & $\begin{array}{l}12.0 \\
12.5\end{array}$ & - & $\begin{array}{l}3.63 \\
2.48\end{array}$ & $\begin{array}{l}0.263 \\
0.126\end{array}$ \\
\hline
\end{tabular}

a Dubious - sea water contaminated?

${ }^{\mathrm{b}}$ Wash cores. 
TABLE 2

Chemistry of in situ Samples

\begin{tabular}{|c|c|c|c|c|c|c|c|c|c|c|}
\hline Sample & $\begin{array}{c}\text { Depth } \\
(\mathrm{m})\end{array}$ & $\mathrm{pH}$ & $\begin{array}{l}\text { Alkalinity } \\
\text { (meq/L) }\end{array}$ & $\begin{array}{c}\text { Salinity } \\
(\%)\end{array}$ & $\underset{(\%)}{C l}$ & $\begin{array}{c}\mathrm{Ca} \\
(\mathrm{mM})\end{array}$ & $\begin{array}{l}\mathrm{Mg}^{\mathrm{a}} \\
(\mathrm{mM})\end{array}$ & $\begin{array}{l}\mathrm{Mg}^{\mathrm{b}} \\
(\mathrm{mM})\end{array}$ & $\begin{array}{l}\mathrm{NH}_{4} \mathrm{c} \\
(\mathrm{mM})\end{array}$ & $\begin{array}{l}\mathrm{SiO}_{2} \mathrm{c} \\
(\mathrm{mM})\end{array}$ \\
\hline \multicolumn{11}{|l|}{ Site 438} \\
\hline 1 & 59 & 8.0 & 11.2 & - & 18.6 & 6.6 & 54.0 & 40.3 & 1.65 & 0.583 \\
\hline 2 & 116 & 7.9 & 23.9 & 34.1 & 17.3 & 6.3 & 35.7 & 29.2 & 3.18 & 0.742 \\
\hline 3 & 164 & 7.7 & 25.1 & 33.1 & 18.6 & 6.6 & 12.6 & 28.0 & - & - \\
\hline 4 & 211 & 7.5 & 23.5 & 32.5 & 18.2 & 9.5 & 16.3 & 26.1 & 3.75 & - \\
\hline 5 & 259 & 7.6 & 12.6 & 32.0 & 17.7 & 9.0 & 11.5 & 28.7 & - & - \\
\hline 6 & 306 & 7.6 & 12.0 & 32.5 & 17.6 & 9.3 & 11.0 & 27.2 & - & - \\
\hline 7 & 354 & 7.6 & 16.9 & 30.5 & 17.2 & 8.2 & 10.6 & 21.8 & - & - \\
\hline 8 & 401 & - & - & - & 16.7 & 8.8 & 24.4 & 17.9 & 3.49 & 0.868 \\
\hline 9 & 449 & - & - & 27.2 & 15.1 & 9.8 & 13.4 & 11.1 & - & - \\
\hline \multicolumn{11}{|l|}{ Site 440} \\
\hline 1 & 64 & 7.8 & 61.1 & 37.0 & 19.2 & 3.3 & 60.4 & - & 4. 18 & 0.115 \\
\hline 2 & 5 & 8.4 & 36.3 & 35.7 & 19.2 & 6.0 & 54.2 & - & 3.56 & 0.421 \\
\hline 3 & 130 & - & - & 38.2 & 19.2 & 2.6 & 61.6 & - & 4.70 & 0.117 \\
\hline 4 & 178 & 7.9 & 83.9 & 37.3 & 19.7 & 2.5 & - & - & 5.10 & 0.122 \\
\hline 5 & 235 & 8.0 & 75.4 & 38.0 & 19.0 & 4.6 & 64.3 & - & 5.03 & 0.154 \\
\hline
\end{tabular}

${ }_{b}^{a}$ Shipboard data-titration.

${ }^{6}$ Stored Cu-tube samples-atomic absorption.

${ }^{c}$ On overflow samples-corrected for dilution; data may be affected by $\mathrm{Cu}^{2+}$ from tubing.
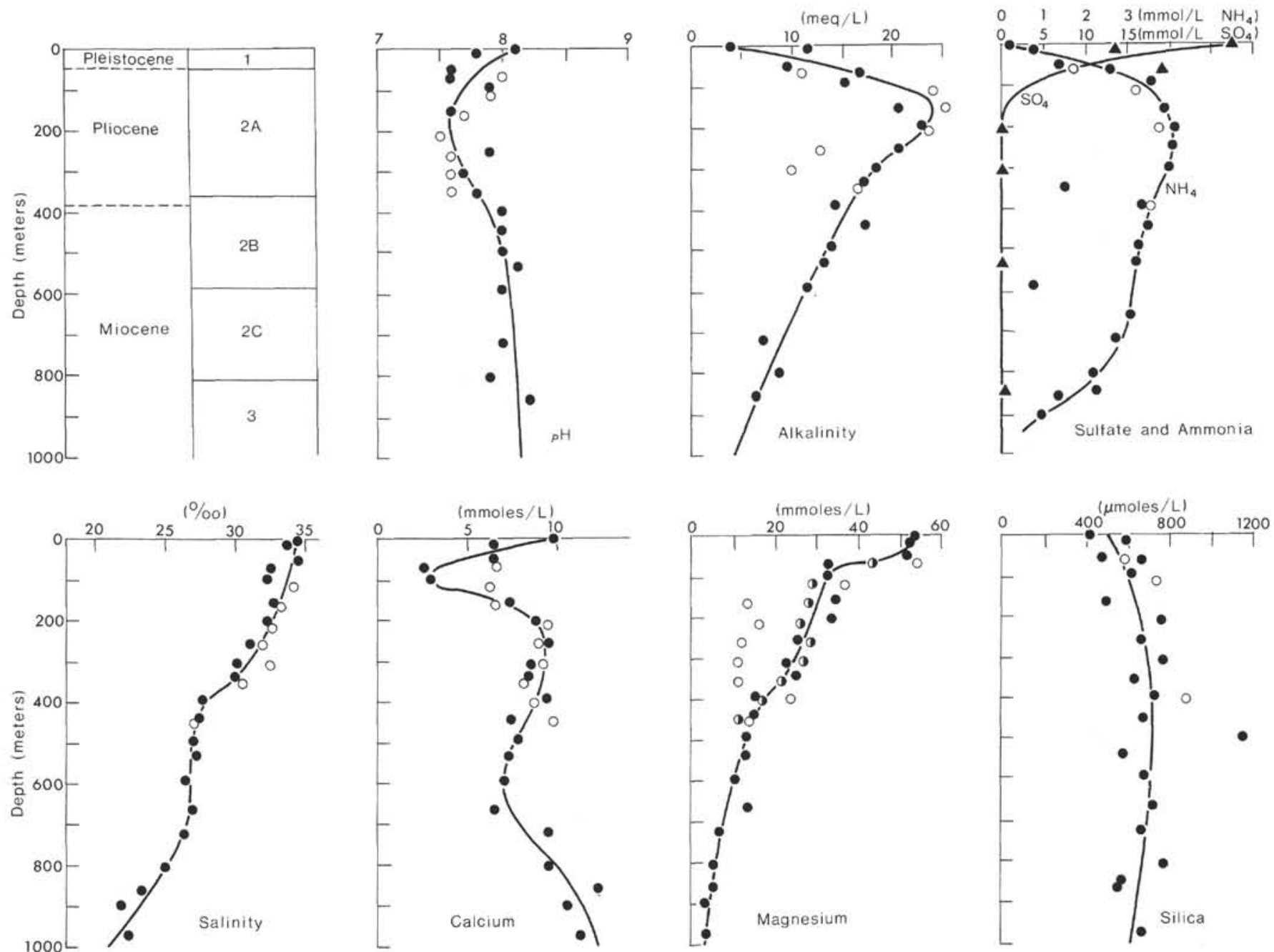

Figure 2. Interstitial water data, DSDP Site 438. Lithology: (1) diatomaceous sandy-silty clay with numerous ash layers; $(2 A)$ clayey diatomaceous ooze, diatomaceous ooze, and diatomaceous clay with numerous ash layers; $(2 B)$ diatomaceous claystone and clayey diatomite; (2C) claystone and diatomaceous claystone; (3) sandy claystone and diatomaceous claystone. Data in open symbols are in situ values; half-filled circles for magnesium are in situ values from stored copper coils. 

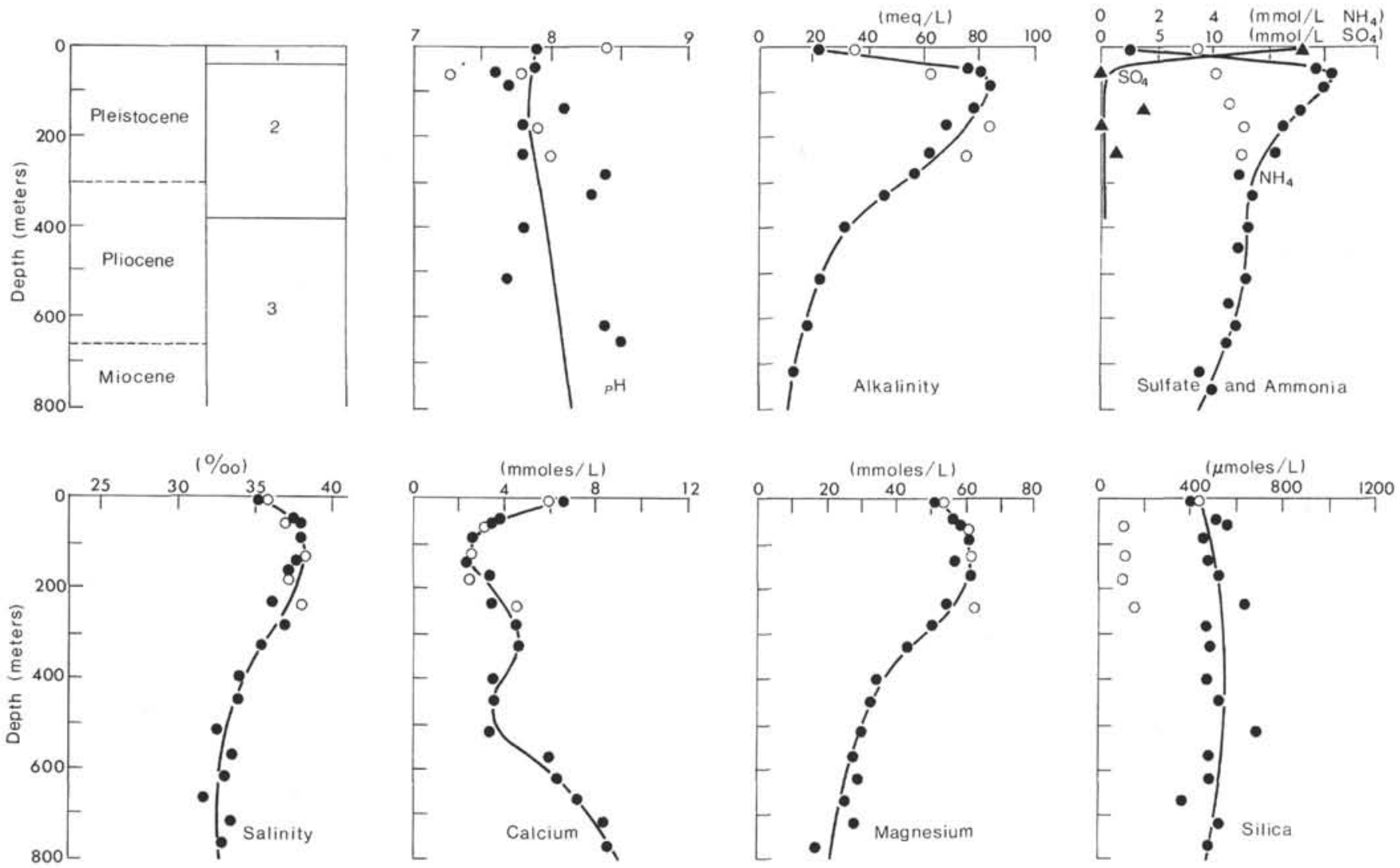

Figure 3. Interstitial water data, DSDP Site 440. Lithology: (1) clayey sand and gravel and diatomaceous clay; (2) diatomaceous clay and claystone (below $168 \mathrm{~m}$ ); (3) interbedded claystone and diatomaceous claystone. Data in open symbols are in situ values.

are relics of a time when those strata were within 200 meters of the sea floor.

\section{SILICA MINERAL TRANSFORMATIONS}

Dissolved silica analyses scatter around $500 \mu \mathrm{M}$, a typical value for material containing both clay minerals and biogenic silica. Diatoms exist to a greater depth in Leg 57 holes than in many other Deep Sea Drilling Project holes because the geothermal gradient is low, especially in the holes on the trench slope, probably because of subduction of cool material below the sites.

At both Site 440 (deepest core $808 \mathrm{~m}$ ) and Site 441 (deepest core $671 \mathrm{~m}$ ) on the trench slope, diatom frustules occur to the base, and we found no secondary silica minerals at these sites by scanning electron microscopy controlled by X-ray fluorescence spectroscopy. At Sites 438 and 439 on the deep sea terrace above the trench slope, the cores first contain embryonic secondary silica lepispheres at a depth of 851 meters (middle Miocene), and they contain secondary quartz below an unconformity at a depth of 1149 meters (Upper Cretaceous).

The diatomaceous strata contain an average of about 15 per cent biogenic silica. The silica lepispheres are about $4 \mu \mathrm{m}$ in diameter (Figure 4), and, as in the case of many other examples found by the Deep Sea Drilling Project, they occur in close association with diatoms, commonly nestled individually within the pores of diatom frustules.

Microscopy shows that the transformation of diatoms into silica lepispheres and then into quartz in the cores of Leg 57 involves dissolution and reprecipitation at each stage. The materials occur in a sequence of decreasing solubility in which each phase provides material to solution so as to form the more stable phase that follows it.

Because the silica mineral phases in diatoms and in silica lepispheres have very small crystallite sizes with respect to the wave length of X-rays, X-ray diffraction spacings are difficult to characterize precisely. Also, crystallite shapes that are systematic with respect to lattice orientations reduce or increase the diffraction from certain spacings.

Diatoms diffract over a broad $d$-spacing range of 4.0 to $4.5 \AA$, and silica lepispheres diffract mainly at $4.1 \AA$, the principal spacing for $\alpha$-cristobalite and the secondary one for $\alpha$-tridymite.

The term opal- $A$ has been applied to diatoms and the term opal-CT to silica lepispheres, and the necessary decrease of solubility within the sequence has been ascribed to lattice disorder and to interlayering of cristobalite and tridymite in opal-CT (Jones and Segnit, 1971). Other investigators have proposed, however, that tridymite may be the only mineral in opal-CT, on the 
TABLE 3

Carbonate Mineralogy, DSDP Leg 57

\begin{tabular}{|c|c|c|c|c|c|}
\hline $\begin{array}{c}\text { Sample } \\
\text { (cm below top of Section) }\end{array}$ & Age & $\begin{array}{l}\text { Depth } \\
(\mathrm{m})\end{array}$ & Lithology & $\begin{array}{l}\text { Carbonate Mineralogy } \\
(\%)\end{array}$ & $\begin{array}{c}\mathrm{MgCO}_{3} \text { in Calcite } \\
\text { (mole \%) }\end{array}$ \\
\hline $438 \mathrm{~A}-66-2(54)$ & Late Miocene & 681 & Limestone breccia, clast & calcite, 100 & 4 \\
\hline $438 \mathrm{~A}-66-2(54)$ & Late Miocene & 681 & Limestone breccia, vein & calcite, 100 & 3 \\
\hline $438 \mathrm{~A}-68-2(17)$ & Middle Miocene & 699 & Limestone & calcite, 100 & 8 \\
\hline $438 \mathrm{~B}-21-4(115)$ & Early Miocene & 1020 & Calcareous concretion & calcite, 100 & 12 \\
\hline $439-5-1(37)$ & Middle Miocene & 850 & Calcareous concretion & calcite, 100 & 8 \\
\hline $439-7-1(108)$ & Early Miocene & 870 & Limestone above tuff bed & calcite, 100 & 9 \\
\hline $439-11, \mathrm{CC}(7)$ & Early Miocene & 912 & Glauconitic limestone, matrix & calcite, 100 & 5 \\
\hline $439-11, \mathrm{CC}(7)$ & Early Miocene & 912 & Glauconitic limestone, vein & calcite, 100 & 2 \\
\hline $439-11-2,(111)$ & Early Miocene & 935 & Limestone & calcite, $80 ;$ ankerite, 20 & 5 \\
\hline $439-24-6(140)$ & Late Oligocene & 1028 & Siltstone & calcite, $60 ;$ ankerite, 40 & 8 \\
\hline $439-30-3(140)$ & Late Oligocene & 1082 & Calcareous silty sandstone & calcite, 40 ; ankerite, 10 ; dolomite, 50 & 3 \\
\hline $440-4-3(90)$ & Late Pleistocene & 29 & Calcareous silty clay & calcite, $60 ;$ ankerite, 40 & 10 \\
\hline $440 A-3-2(145)$ & Late Pleistocene & 95 & Calcareous sandy silty clay & calcite, 100 & 1 \\
\hline $440 \mathrm{~A}-6-2(93)$ & Early Pleistocene & 123 & Calcareous clayey diatom ooze & calcite, 100 & 1 \\
\hline $440 B-9-4(13)$ & Early Pleistocene & 220 & Chalk & calcite, 50 ; ankerite, 50 & 5 \\
\hline $440 B-15-1(44)$ & Early Pleistocene & 273 & Chalk & calcite, $80 ;$ ankerite, 20 & 5 \\
\hline $440 \mathrm{~B}-22-3(125)$ & Late Pliocene & 343 & Chalk & calcite, 60 ; ankerite, 40 & 3 \\
\hline $440 B-64-1(120)$ & Late Miocene & 739 & Limestone & calcite, 100 & 4 \\
\hline $441-5, \mathrm{CC}(0)$ & Late Pliocene & 83 & Concretionary pebble & calcite, 70 ; ankerite, 30 & 6 \\
\hline $441 \mathrm{~A}-2, \mathrm{CC}(10)$ & Late Pliocene & 130 & Calcareous concretion & calcite, 100 & 4 \\
\hline $441 \mathrm{~A}-9-1$ & Early Pliocene & 568 & Calcareous concretion & calcite, 100 & 5 \\
\hline
\end{tabular}

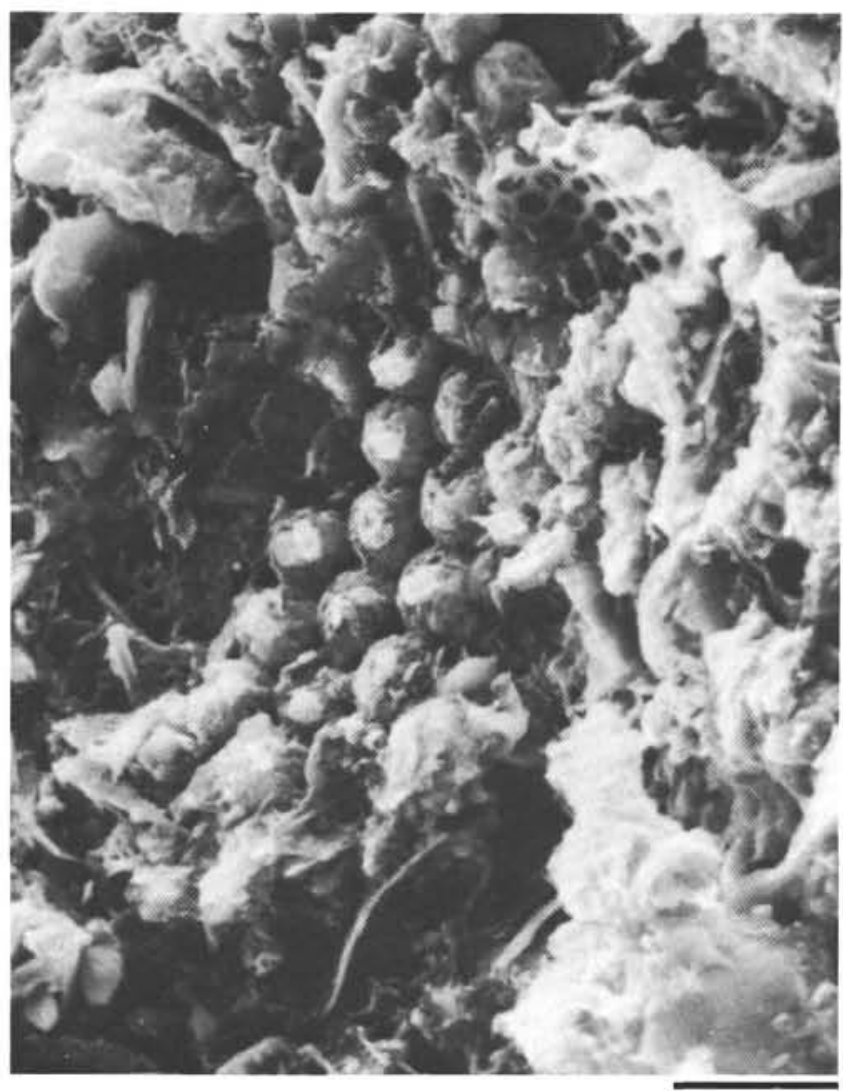

$10 \mu \mathrm{m}$

Figure 4. Silica lepispheres at a depth of 851 meters, DSDP Site 438.

basis of its infrared spectrum and the fact that especially large crystallites on the surfaces of some lepispheres have been characterized by electron diffraction as tridymite (Wilson et al., 1974).
The decreasing order of solubility of the minerals is cristobalite, tridymite, and quartz (Tuttle and England, 1955; Fournier, 1973). Hence if diatoms are composed of extremely fine-grained cristobalite and if silica lepispheres are composed of tridymite, a rational sequence results. The X-ray diffraction related to tridymite's main $d$-spacing of $4.3 \AA$ may be relatively suppressed over that of $4.1 \AA$, which tridymite shares with cristobalite, because systematically oriented slender radial pyramidal crystallites in the lepispheres are elongated so as to enhance diffraction related to the spacing of $4.1 \AA$.

The rate of transformation of diatoms into silica lepispheres near the Japan Trench matches well with that ascertained by Hein and others (1978) in the Bering Sea. A natural temperature of $32{ }^{\circ} \mathrm{C}$ measured at a depth of 862 meters below the sea floor in Hole 438A (Figure 5), when combined with a sea floor temperature of $1^{\circ} \mathrm{C}$, gives an average geothermal gradient of $3.6^{\circ} \mathrm{C} / 100 \mathrm{~m}$. Hence the natural temperature of the strata at 851 meters containing the highest lepispheres at Site 438 is $31^{\circ} \mathrm{C}$. When combined with the 16-Ma age of the strata, this point plots very close to a diatom to lepisphere kinetic boundary based on a least-squares average of the data presented by Hein and others (1978). A graph of geologic age to temperature for the cores at Sites 438 and 439 (Figure 6) illustrates how the cores range from the diatom, through the silica-lepisphere, to the quartz fields. Hence the data from Leg 57 confirm the accuracy of this silica geothermometer in reconstructing the temperature history of sequences of sedimentary rocks that contain siliceous microfossils.

\section{ACKNOWLEDGMENTS}

John Rutherford carried out the shipboard chemical analyses reported in this paper. James L. Bischoff and Caroline M. Isaacs reviewed the manuscript. 


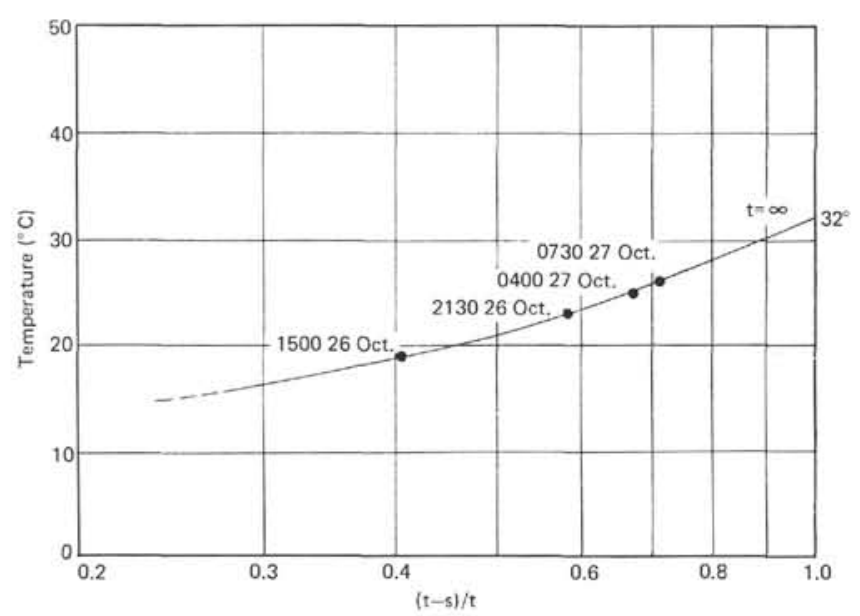

Figure 5. Equilibrium temperature of $32^{\circ} \mathrm{C}$ at a depth below the sea floor of 862 meters in Hole 438A, based on temperature measurements at several times $\mathrm{t}$ after that depth was drilled at 230025 October 1977 and on the time s $(9.5 \mathrm{hr})$ from the drilling until circulation of the drilling fluid ceased at 0830 October (Lachenbruch et al., 1966).

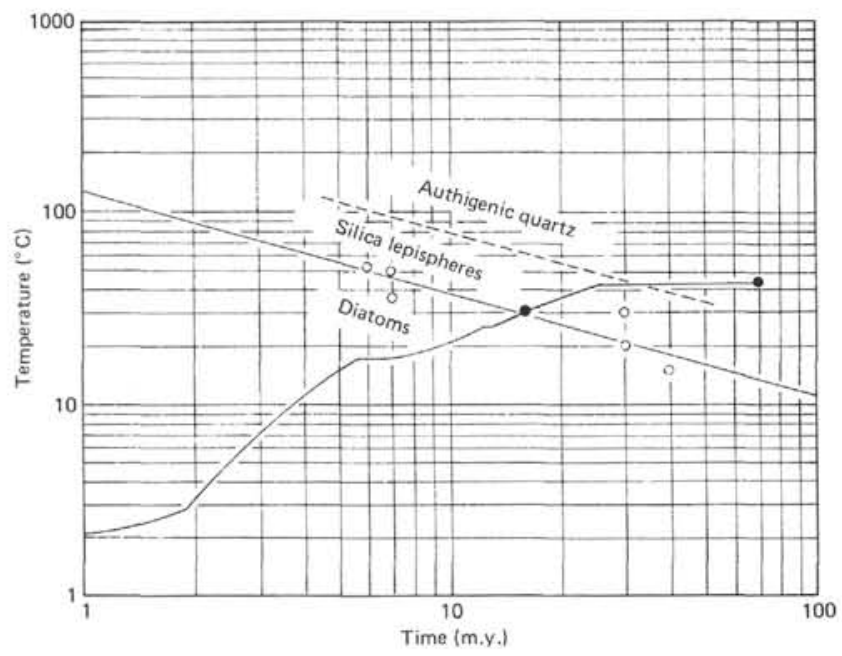

Figure 6. Relationship between geologic age, temperature in place, and silica phases for cores from DSDP Sites 438 and 439. Open circle diatom to lepisphere data points after Hein and others (1978). The two solid circles on the irregular line representing the cores mark the shallowest silica lepispheres and authigenic quartz, respectively.

\section{REFERENCES}

Barnes, R. O., Gieskes, J. M., Horvath, J., and Akiyama, W., 1979. Interstitial water studies, Legs 47A, B. In Ryan, W. B. F., Sibuet, J.-C., et al., Init. Repts. DSDP, 47, Pt. 2: Washington (U.S. Govt. Printing Office), 577-582.

Boles, J. R., 1978. Active ankerite cementation in the subsurface Eocene of southwest Texas. Contrib. Mineral. Petrol., $68,13-22$.

Cook, P. J., 1974. Geochemistry and diagenesis of interstitial fluids and associated calcareous oozes, Deep Sea Drilling Project, Leg 27, Site 262, Timor Trough. In Veevers, J. J., Heirtzler, J. R., et al., Init. Repts. DSDP, 27: Washington (U.S. Govt. Printing Office), 463-480.

Fournier, R. O., 1973. Silica in thermal waters: Laboratory and field investigations. In Clarke, J. W. (Ed.), Proceedings of Symposium on Hydrogeochemistry and Biogeochemistry: Washington (Clarke), pp. 122-139.

Gieskes, J. M., 1974. Interstitial water studies, Leg 25 . In Simpson, E. S. W., Schlich, R., et al., Init. Repts. DSDP, 25: Washington (U.S. Govt. Printing Office), 361-394. 1975. Chemistry of interstitial waters of marine sediments. Ann. Rev. Earth Planet. Sci., 3, 433-453.

Hartmann, M., Muller, P., Suess, E., and van der Weyden, C. H., 1973. Oxidation of organic matter in recent marine sediments. 'Meteor'” Fortschungsergeb., Ser. C., No. 12, 7486.

Hein, J. R., Scholl, D. W., Barron, J. A., Jones, M. G., and Miller, J., 1978. Diagenesis of late Cenozoic diatomaceous deposits and formation of the bottom simulating reflector in the southern Bering Sea. Sedimentology, 25, 155-181.

Honza, E., Tamaki, K., and Murakami, F., 1978. Geological map of the Japan and Kuril Trenches and the adjacent areas. Geological Survey of Japan Marine Geology Map Series, No. 11, scale 1:1,000,000.

Johnson, T. C., 1976. Biogenic opal preservation in pelagic sediments of a small area in the eastern tropical Pacific. Bull. Geol. Soc. Am., 87, 1273-1282.

Jones, J. B., and Segnit, E. R., 1971. The nature of opal: I. Nomenclature and constituent phases. J. Geol. Soc. Aust., $18,57-68$,

Lachenbruch, A. H., Greene, G. W., and Marshall, B. V., 1966. Permafrost and the geothermal regimes. In Wilimovsky, N. J., and Wolfe, J. N. (Eds.), Environment of the Cape Thompson Region, Alaska: Oak Ridge (U.S. Atomic Energy Commission), pp. 149-163.

Sayles, F. L., and Mangelsdorf, P. C., Jr., 1977. The equilibration of clay minerals with seawater. Geochim. Cosmochim. Acta, 41, 951-960.

Soong, R., and Gluskoter, H. J., 1977. Mineral matter in some New Zealand coals. New Zealand J. Sci., 20, 273-277.

Tuttle, O. F., and England, J. L., 1955. Preliminary report on the system $\mathrm{SiO}_{2}-\mathrm{H}_{2}$ O. Bull. Geol. Soc. Am., 66, 149-152.

Wilson, M. J., Russell, J. D., and Tait, J. M., 1974. A new interpretation of the structure of disordered $\alpha$-cristobalite. Contrib. Mineral. Petrol., 47, 1-6. 